\title{
Las lesiones de tronco de coronaria izquierda: ¿Cirugía o intervencionismo?.
}

\author{
ASTE SALAZAR Hernán*
}

SUMMARY:

In recent years there has been some improvement in surgical and interventional techniques that have modified the classical indications making them more risky. Left main coronary stenoses are even more dreadful lesions, and the results of percutaneous management are odd with varying mortality (10-12\% on average), and high morbidity. Moreover we have small number of cases in the literature. Comparing these results with surgery, we find the later to be safer with mortality under $4 \%$, low morbidity and a long term experience in the management of this pathology. Results, moreover, tend to favor surgery in the coronary restenosis in spite of the use of stents. (Rev Med Hered 2003; 14: 204-206).

\section{KEY WORDS: Coronary stenoses, coronary restenosis}

En la evolución de la medicina, los procedimientos destinados a lograr objetivos terapéuticos suelen pasar por una serie de etapas conocidas como curva de "Performance" o "Desempeño" del procedimiento relacionándolo en el tiempo. Van desde la idea o hipótesis hasta la definición final con una evaluación de su eficiencia (Figura $\mathrm{N}^{\circ} 1$ ).

Las lesiones de tronco de coronaria izquierda (TCI) conforman el 0.7 al $12.6 \%$ de los revascularizados, y las lesiones aisladas de tronco el 3 al $6 \%$ de esta patología (1). Las lesiones ostiales hacen el $17.9 \%$ de las lesiones de tronco, siendo más frecuentes en mujeres que en varones.

El manejo médico conservador en lesión de TCI $>50 \%$ diagnosticada es un error si se cuenta con cirugía, ya que la mortalidad anual del manejo médico es de $19 \%$ y la sobrevida a 3 años está alrededor de $50 \%$ (1). En el trabajo reciente de Hemingway (2), et al. se demostró el beneficio de operar a los pacientes que tienen indicación clara e inclusive dudosa de cirugía con respecto a los manejados conservadoramente e inclusive con intervencionismo.
El intervencionismo ha mejorado gradualmente en los últimos tiempos no llegando a superar a la cirugía, sin embargo, la "reestenosis" aún no se puede eliminar a pesar del uso de stents. Los avances en los procedimientos percutáneos han sido importantes: mejores balones, mejores stents, guías más navegables, el uso del ultrasonido intravascular, la aparición de los antagonistas de la glicoproteína IIbIIIa; una ganancia en experiencia y la lucha por eliminar la reestenosis sea con braquiterapia y/o los

Figura $\mathrm{N}^{\circ} 1$ Curva de Performance de un procedimiento con tendencia a ser favorable.

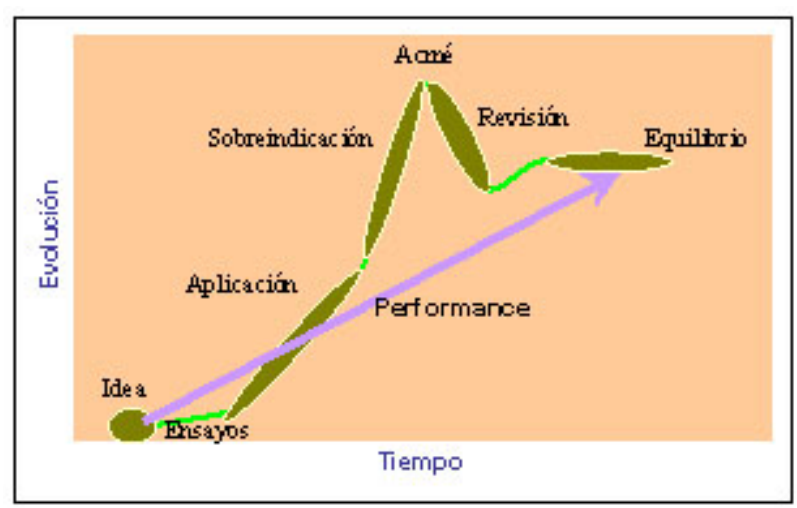

* Médico Cirujano Cardiovascular

Cirujano Titular de la Clínica San Borja, Lima-Perú 
stents recubiertos con rapamicina (Sirolimus) que está dando mejores resultados hasta el momento lo que ha estimulado a los hemodinamistas a ser más osados. No todas las angioplastías son iguales, ya que existen las angioplastías llamadas de "alto riesgo" (incluyen las lesiones de tronco y de ostium de Coronaria izquierda) (Figura $\mathrm{N}^{\circ} 2$ ) en las cuales las posibilidades de complicación y las mismas complicaciones son significativas(3): Disfunción ventricular severa, alta probabilidad de reoclusión durante o tempranamente a una angioplastía, y alta probabilidad de compromiso hemodinámico durante el inflado del balón.

En los últimos tres años han aparecido una serie de publicaciones que se refieren al intervencionismo de TCI y todos ellos hacen gala de tratamiento percutáneo sobre este territorio en lo que se conoce como "TCI no protegido" (Unprotected Left Main Coronary Artery) como un TCI que no tiene un puente patente a descendente anterior o a circunfleja, sin considerar la circulación inter o intracoronaria.

En artículos representativos recientes la casuística sumada no llega a 700 pacientes, el trabajo que más casuística presenta tiene un máximo de 279 pacientes, por tanto la cantidad de casos es pequeña. Los casos incluidos, en 3 de los 5 trabajos presentados, comparan a los que serían buenos candidatos para cirugía y los malos candidatos (v.g.: disfunción ventricular, infarto agudo de miocardio [IMA], shock cardiogénico, edad muy avanzada, Enfermedad pulmonar obstructiva crónica, etc). En 2 de ellos la condición era isquemia documentada en uno e IMA en el otro. La fracción de eyección (FE) en la mayoría era superior al $40 \%$ a excepción del trabajo de Hon-Kan (pacientes con IMA) en cuyo caso la FE era variable inclusive algunos con severa disfunción. Asimismo, la mayoría era electivo para el procedimiento.

\section{Figura $\mathbf{N}^{\circ}$ 2. Lesión de ostium (flecha) y tronco de CI por una aortitis.}

\section{A) Pieza patológica de lesión.}

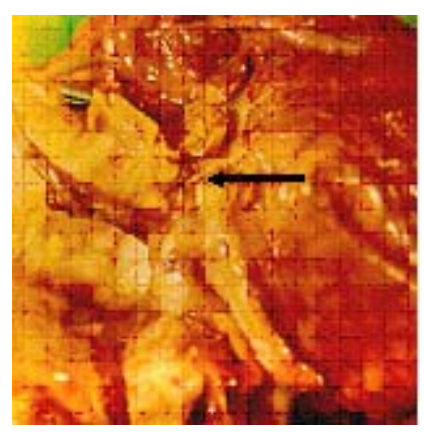

B) Angiografía.

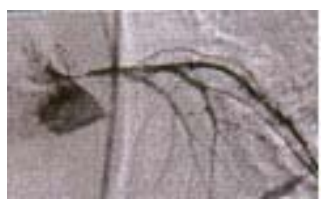

Los resultados inmediatos y del seguimiento (15 a 20 meses) de los pacientes estudiados fueron: la mortalidad temprana en el trabajo de Ellis $12.1 \%$ de muertes, pero si el paciente estaba en IMA la mortalidad era $69 \%$, con mortalidad global de 29\%. Hon-Kan tuvo 33\%, con mortalidad global fue 44\%. En los resultados de Black, Park y Silvestri el promedio de mortalidad está alrededor de $7 \%$ tanto en forma temprana como en la global; en ellos los casos fueron electivos todos, con buena FE, no IMA, etc.

En 1998 el Dr. René Favaloro (4) en una revisión detallada de la evolución de la cirugía desde 1967 hasta 1997, revisa el origen, los progresos, los trabajos comparativos con tratamiento médico y angioplastía, y algunos alcances con el uso de stents. En 1999 aparece la actualización de las Guías de la American Heart Association (AHA) y el American College of Cardiology (ACC) sobre la Cirugía de Coronarias (5). En ambos trabajos las lesiones de TCI son indicación primaria para revascularización quirúrgica. Desde 1999 hasta el 2002 en algunos centros se ha procedido a dilatar y colocar stents en tronco de coronaria izquierda con resultados controversiales. En las revisiones de cirugía los resultados obtenidos han sido bastante buenos siempre, con mortalidad menor al $4 \%$ y reintervenciones escasas, en cifras recientes, y así como el intervencionismo mejoró en los últimos años, la cirugía también ha mostrado avances: mejor protección miocárdica, mejores agentes anestésicos y técnicas de anestesia, mejor analgesia post operatoria, y el manejo en general; se cuenta con cirugía sin bomba (6) (sin circulación extracorpórea), la cirugía mínimamente invasiva, el uso de conductos arteriales e inclusive la robótica; a todo esto se suma una experiencia mundial de más de 30 años siendo uno de lo procedimientos quirúrgicos más analizados en medicina y contando con la base de datos voluntaria cardiológica de adultos más grande en medicina en manos de la Sociedad de Cirujanos Torácicos (STS) de los Estados Unidos (7).

En algunas circunstancias se pueden combinar las estrategias realizando una angioplastía para estabilizar el cuadro (como puente) para luego programar, de inmediato, la corrección quirúrgica. La angioplastía, además, tiene un índice de complicaciones como las oclusiones agudas, los hematomas intramurales (8), las disecciones, las perforaciones; $\mathrm{y}$ sabemos que hay hasta un $8 \%$ de fallos en las angioplastías a pesar del desarrollo del stent $(9,10)$, que en lesión de tronco puede ser fatal. Es más, la mortalidad operatoria es mayor cuando una cirugía se hace sobre una angioplastía fallida (>10\%), y si el paciente requiere maniobras de resucitación en su camino a sala de operaciones, ésta se eleva hasta $45 \%$. 
La pregunta es: y ¿en qué parte de la curva de Performance se encuentran la Cirugía de lesión de tronco y la angioplastía del mismo? ....la cirugía sigue siendo el "estándar de oro".

\section{Correspondencia:}

Hernán Aste Salazar

Av. Guardia Civil 333 San Borja

Lima Perú

e-mail: aste@amauta.rcp.net.pe

\section{REFERENCIAS BIBLIOGRAFICAS}

1. D'Allonnes FR, Corbineau H, Le Breton H, et al. Isolated left main coronary artery stenosis: Long term follow-up in 106 patients after surgery. Heart 2002; 87:544-8.

2. Hemingway H, Crook AM, Feder G, et al. Underuse of coronary revascularization procedures in patients considered appropriate candidates for revascularization. N Engl J Med 2001; 344: 645-54.

3. Angelini P, Hernández C, Ferguson III JJ, et al. High risk coronary angioplasty assisted by active hemoperfusion. Tex Heart Inst J 1996; 23: 15-23.

4. Favaloro R. Critical Análisis of coronary artery bypass graft surgery: A 30-year Journey. J Am Coll Cardiol 1998; 31 (Suppl B): 1B-63B.

5. Eagle, Guyton, et al. Guidelines for coronary artery bypass graft surgery. J Am Coll Cardiol 1999; 34: 1262-347.

6. Dewey TM, Magee MJ, Edgerton JR, et al. Off-Pump bypass grafting is safe in patients with left main coronary disease. Ann Thorac Surg 2001; 72: 788-92.

7. Ferguson TB, HammillBG, Peterson ED, et al. A decade of change-risk profiles and outcomes for isolated coronary bypass grafting procedures, 1990-1999: A report from de STS National Database Committee and the Duke Clinical Research Institute. Ann Thorac Surg 2002; 73: 480-90.

8. Machara A, Mintz G, Bui A, et al. Incidence, morphology, angiographic findings and outcomes of intramural hematomas after percutaneous coronary interventions. an intravascular ultrasound study Circulation 2002; 105: 2037-42.

9. Craver JM, Justicz AG, Weintraub WS, et al. Coronary artery bypass grafting in patients after failure of intracoronary stenting. Ann Thorac Surg 1995; 60: 606.

10.Ladowski JS, Dillon TA, Deschner WP, et al. Durability of emergency coronary artery bypass for complications of failed angioplasty. Cardiovasc Surg 1996; 4: 23-7. 